\title{
Practices of Stigmergy in Architectural Work
}

\author{
Lars Rune Christensen \\ IT University of Copenhagen \\ Rued Langgaards vej 7 \\ 2300 Copenhagen S, Denmark \\ +4529264538 \\ Lrc@itu.dk
}

\begin{abstract}
Actors coordinate their cooperative efforts by acting on the evidence of work previously accomplished. The paper introduces, on the basis of a field study, the concept of stigmergy to the analysis of coordinative practices in architectural work. It distinguishes between practices of stigmergy and articulation work. Stigmergy is understood as coordination achieved by acting directly on the evidence of work previously accomplished and articulation work is understood as second order efforts to coordinate collaborative work. Furthermore, this leads to a distinction between representational artifacts associated with practices of stigmergy and coordination mechanisms in the service of articulation work.
\end{abstract}

\section{Categories and Subject Descriptors}

K.4.3 [Organizational Impacts]: Computer-supported collaborative work

\section{General Terms}

Design, Human Factors, Theory

\section{Keywords}

Coordination, cooperative work, stigmergy, articulation work, architectural work.

\section{INTRODUCTION}

In this article, an attempt is made to achieve a better understanding of how actors perform the coordination of collaborative work by the use of artifacts. Whereas a number of previous studies have focused on articulation work carried out by using material artifacts (e.g. [17, 18, 2]), the interest here is to explore how actors simply act on evidence of work previously accomplished and how this leads to the coordination of cooperative work. What is implied is a distinction between on the one hand articulation work understood as a 'kind of supra-type work in any division of labour, done by the various actors' concerning the integration of interdependences in cooperative work [23] and on the other hand stigmergy understood as the coordination of cooperative

(C) ACM, (2007). This is the author's version of the work. It is posted here by permission of $\mathrm{ACM}$ for your personal use. Not for redistribution. work based on the evidence of work previously accomplished [11]. We shall return to this conceptual distinction below.

The arguments are based on an ethnographic study of an architectural office which is arguably an excellent location for the study of coordinative practices. Architects and construction engineers at the office continuously shift between face-to-face cooperation and distributed cooperation where they not only rely on articulation work but also on coordination achieved by acting directly on the evidence of work previously accomplished. One of the characteristics of architectural work is the effort to achieve continuity in regard to the design and construction work carried out by different staff members over the course of any given building project. These efforts can take the form of coordinative practices.

The aim is to approach an understanding of how the actors involved in a building project in the design stage coordinate their collaborative work, characterised by a mix of co-located and distributed work, by acting on the evidence of work previously accomplished by themselves or others. The hope is that this will further an understanding of coordination of collaborative work and the design of computer support for such work.

We will proceed in the following manner. First, we will attempt to show, in the context of architectural work, how the participants in a building project at times coordinate their work by acting on the evidence of work previously accomplished. Secondly, in order to further an understanding of such coordinative practices the concept of stigmergy [11] will be introduced and discussed in relation to the concept of articulation work $[23,19]$ and the concept of feedthrough $[6$, 7]. Finally, an outline will be drawn of some of the discussion's perspectives for the field of $\mathrm{CSCW}$ and group work.

\section{METHODS}

The paper is based on fieldwork carried out in the course of five months in an architectural office. In this period, the architectural work on several building projects was studied. One of them, the development of a retirement home in Copenhagen, was a one-storey addition made to an existing multi-story complex. In addition to this project, the construction of sanitation and ventilation in connection to a large building project was studied. A combination of 
observation and interviews was used. The fieldwork also included collecting (scanning, taking screenshots or photographs of) artifacts used and produced by the employees at the architectural office.

\section{ARCHITECTURAL WORK}

As a profession, architecture has emerged and evolved over the past centuries as a discipline mainly concerned with the task of designing buildings, and providing representations of these building designs in the form of schematics and drawings that are used by not least the engineers and craftsmen that do the actual building [25]. Modern architects have to work with a large range of demands and issues that previous generations of architects did not have to handle. During the industrial revolution, the needs of industry and commerce led to the introduction of facilities for heating, ventilation and sanitation, which was taken up in domestic architecture as well. Subsequently, building elements such as elevators, telephones, and mechanical ventilation were introduced in the last decades of the nineteenth century. In the course of the last century, the architecture of modern buildings has been further enriched by the introduction of air-conditioning systems, facilities for telecommunications, security, safety, firefighting, etc [18].

In a standard large building project various actors work on different sections of the building and they may be responsible for particular design tasks. It is characteristic of an ordinary workday inside an architectural workplace that efforts proceed calmly, with little moving about, a mold now and then broken up by bursts of discussion and conversation. At times, the employees will convene for an internal project meeting or for a meeting with an external consultant. At intervals, too, regularly scheduled meetings are held in which all project members take part; here they evaluate the progress of work, fix dates, settle responsibilities, redistribute tasks (if required), and have a discussion about common issues such as design changes. Participants leave the gathering with some general understanding of the progression of the work, with clarifications of what to do and what to be aware of, open issues, dates, and deadlines. Outside the internal project team, a building project involves many external actors: technical specialists for construction, electricity, heating and ventilation etc. Furthermore, several other actors could be involved, including a client and perhaps one or several users, several authorities, building companies, contractors, and sometimes, depending on the size of the project and the contract, a general contractor. Integrating these many professional competencies and their perspectives, mobilizing their support, is a major task that requires careful planning and ongoing communication [18, p.355].

For the design stage of a building project a diverse work ensemble is formed. It is a work arrangement with a division of labor that reflects both the demand of modern buildings and the competences of the work ensemble.

\subsection{CAD in the Design Process}

In the design stage a large range of representational artifacts are employed. These include Computer Aided Design (CAD) models, that are precise and highly detailed, and other artifacts such as sketches, hand-written notes, images, and models that are more informal, imaginative and open. Others again work with scale models, working out their designs by experimenting with different spatial configurations. Here we shall focus especially on the use of CAD models for coordinative purposes.

In the last 30 years or so, computers have become a prominent tool in the design process, and we speak of Computer Aided Design (CAD) in the context of architectural work and construction. Architects, engineers and other specialists create, share and exchange CAD files in order to be able to coordinate their cooperative efforts in regard to designing a building. The files are shared by the use of a repository on a server that is accessed with a web browser such as Firefox or Internet Explorer. Here the latest versions of the CAD files pertaining to the design of the building are stored and accessed by all the members of the work ensemble. The sharing of CAD files is a crucial resource for coordination of the design process, as we shall see below.

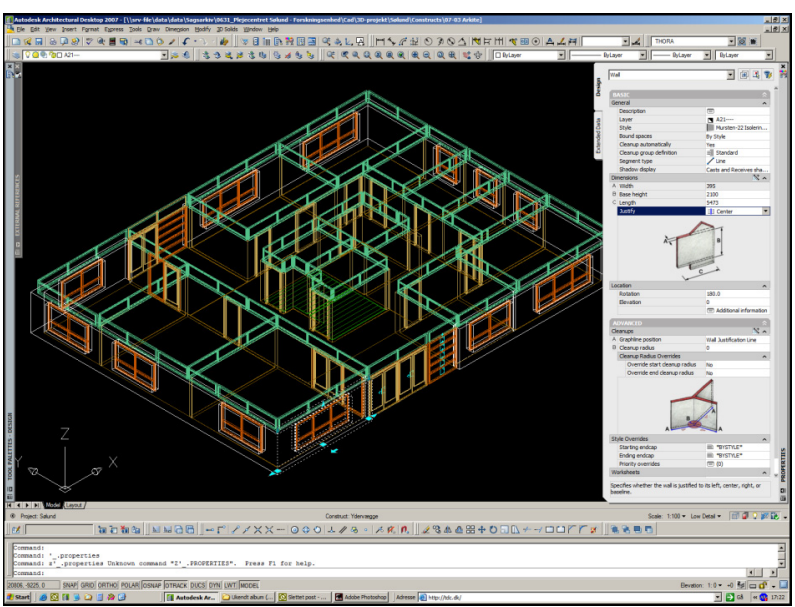

Figure 1: CAD model of a building in three dimensions.

Recently it has become commonplace to design buildings with the use of CAD models that capture the three-dimensional aspects of buildings (see figure 1.). The perception of three dimensions on a computer monitor ${ }^{1}$ is ensured with the help of interactive computer graphics that frees the users from the constraints of a single imposed two-dimensional visual perspective by allowing user controlled rotation and manipulation of the images. It calls upon various techniques, such as rotation, shading, stereoscopy and occlusion to ensure the perception of three dimensions [9, p.402].

Seen from the trajectory of a building project, design and construction is primarily done by the use of CAD models. That is to say, in a modern architectural office, the central representational artifact is the system of CAD models. They incorporate, as an ensemble, a project's trajectory from draft to implementation; they absorb and reflect all decisions taken and changes made, as models are gradually modified and rendered more detailed.

Initially the CAD models constitute the field of work for the ensemble involved in the design process, including architects,

1 The screen of a computer monitor is undoubtedly a twodimensional surface. 
engineers and other specialists. Subsequently the CAD models are used as an indispensable resource for the craftsmen and engineers actually erecting the building. It is the former design process that we are concerned with here.

As mentioned above, actors meet on a regular basis face-toface or over the telephone in order to discuss the 'big picture', the overall progress of the project, who does what when etc. However, when it comes to the coordination of the 'small things' the multitude of details involved in designing a building, this is done not in meetings or over the phone (there is no time), but directly with the CAD models exchanged by the use of the repository. That is, on the detail level of the CAD models, work is primarily coordinated through the work itself, rather than through discourse about it. Perhaps we could take a look at how this process of design with CAD models unfolds.

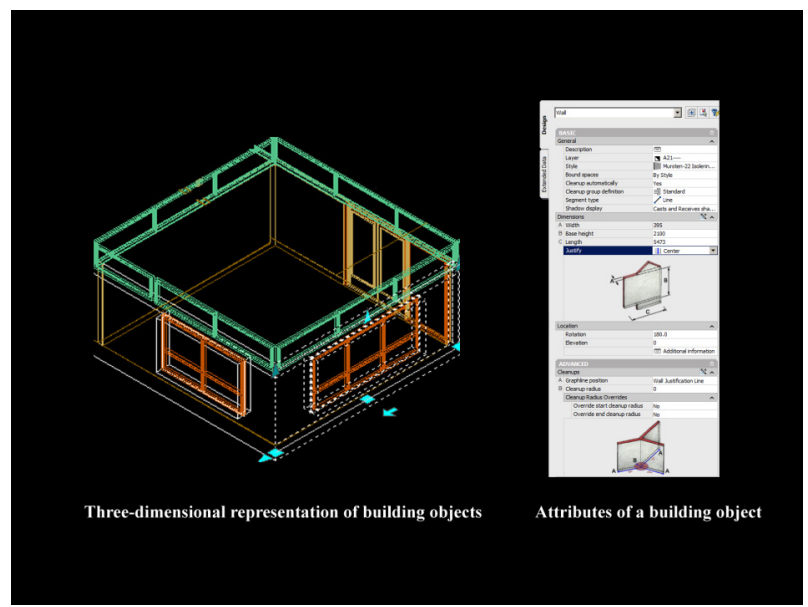

Figure 2: Building objects and attributes in a CAD model.

The basic units in any CAD model of a building are referred to as a 'building objects' (see figure 2.). Building objects commonly used are representations of doors, windows, ceiling, staircase etc. A building object such as a wall, for example, contains geometry as well as specifications of its attributes. In most cases these building objects are predefined in the database of the CAD application, serving as a resource for the architect or construction engineer. The construction engineer, for example, creates the construction model by combining and manipulating a number of building objects - he or she could for example combine floor objects with ceiling objects and wall objects. At a later date, the same or perhaps another actor could add window objects and door objects to the model in a manner consistent with the placement of the previously placed building objects. That is to say, the individual actor creates part of a CAD model by combining a number of conventional predefined building objects. In turn the same or perhaps another actor notices this and ads further building objects to the CAD model.

In this manner the work ensemble, including architects, engineers, specialists etc., all make distinct contributions in the form of models covering their respective areas of expertise. The architect creates the outline of the building. On that basis the construction engineer creates the geometry of the concrete structure in a separate construction model. Subsequently, the sanitation specialist, for example, will take notice of the model for the concrete structure and seek to align the sanitation with it. In a similar manner, the electricity specialist, for example, will take notice of the previously created models and seek to align the wiring of the electricity with it. That is to say, the individual actor creates and changes the form of a CAD model, not for the purpose of conveying a message, but simply as a part of constructing a building; another actor takes notice and acts upon it. In this manner, components of the building such as concrete structure, sanitation, ventilation and electricity are brought into alignment with the overall design (see figure 3.).

The actors are simply doing their job, going about their business without making any second order effort to coordinate anything, and yet coordination of collaborative work is taking place. Cooperative work is coordinated by virtue of the evidence found on artifacts of work previously accomplished.

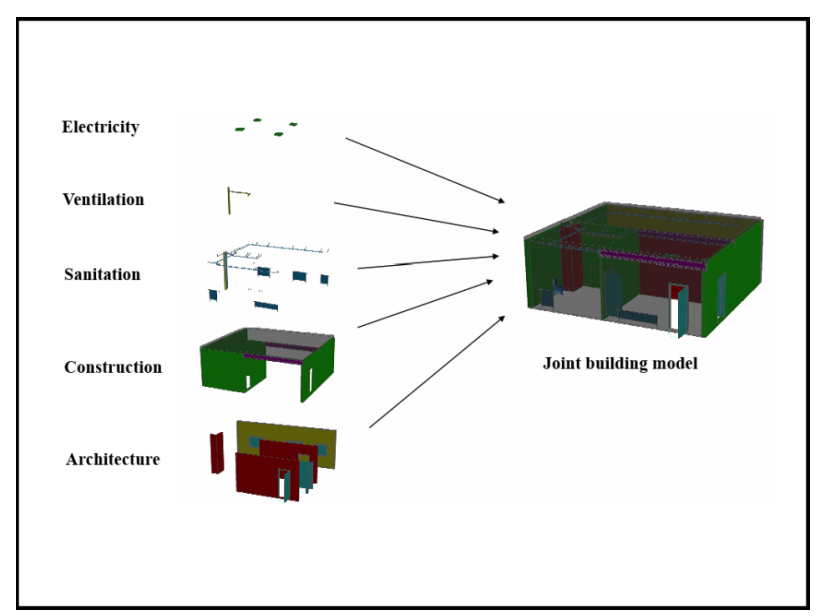

Figure 3: The joining of a number of specialists CAD models into a joint model of a building.

That is to say, in addition to relying on meetings, plans and schedules, actors coordinate their cooperative efforts by acting directly on the evidence of work previously accomplished by themselves or others. This notion of coordination, or rather how to conceptualise it, shall be our concern for the remainder of this paper.

\section{STIGMERGY, ARTICULATION WORK AND FEEDTHROUGH}

As mentioned above, one of the major research issues in $\mathrm{CSCW}$ is the understanding of how cooperative work is coordinated and integrated by using artifacts. This issue has often been cast as a question of exploring how articulation work is practiced and supported by way of artifacts. A series of focused, in-depth field studies have been undertaken with the specific purpose of investigating how the distributed activities of cooperative work arrangements are articulated and, in particular, how prescribed artifacts are devised, appropriated and used for these purposes (e.g. [19, 4, 5, 17, 22]).

These studies have provided invaluable insights. Perhaps, however, it could be fruitful to complement the concept of 'articulation work' with a supplementary means of describing how cooperative work is coordinated and integrated by the use of artifacts. 
In the words of Strauss [23, p.8], articulation work is a kind of supra-type work in any division of labour, done by the various actors concerning the meshing and integration of interdependencies inherent in cooperative work. The prefix 'supra-' is of key importance here ${ }^{2}$. In the context of cooperative work this could entail that articulation work comes before or stands in a meta-relationship to a work task or a set of work tasks performed. We could suggest that the distinction between the articulation work and the cooperative work articulated is an inherent feature of the concept of articulation work. Articulation work in the context of architectural practice often revolves around meetings where the progress of work is discussed, dates are settled, responsibilities cleared up, and work tasks are distributed and redistributed (if need be). These coordinative efforts are often done with the support of what Schmidt \& Simone have described as coordination mechanisms such as work schedules, plans and forms [17]. These observations concerning the second order nature of articulation work are hardly controversial.

Recall the coordinative practices described above: An actor changes the form of a geometrical representation of a building in a CAD model, not for the purpose of conveying a message, but simple as a part of constructing a building; another actor notices this, and in turn acts upon this change of state. In more abstract terms we could say: Signs left or modifications made by individuals on artifacts may, given an appropriate context of practice, become meaningful to these individuals themselves or to others and in turn inspire new actions on artifacts.

How could we describe practices of this nature? Probably not in terms of articulation work, bearing in mind that articulation work refers to an activity that could be described as a second order effort to coordinate collaborative work. In the above example no such second order effort is found, no supra-type relationship is apparent, no meta-discourse about the work at hand is to be found. The actors are simply doing their job, going about their business without making any second order effort to coordinate anything, and yet coordination of the design of the building is taking place.

\subsection{The concept of Stigmergy}

Perhaps we could use the concept of stigmergy to complement our descriptions of coordinative practices. The concept of stigmergy was not developed in order to describe human practice [11]. Rather, it was developed to tackle problems pertaining to the field of entomology. Grassé coined the concept during his study of termite behaviour [24, p.97]. When looking at a group of termites, they all seem to cooperate in building nests etc., but when looking at single individuals they seem to be working as if they were alone and not involved in any collective behaviour. This appeared to be a paradox until Grassé introduced the concept of stigmergy ${ }^{3}$. Grassé showed

2 According to the Oxford dictionary 'supra-' designates a prefix used in describing a phenomenon that is transcending, before or above something else.

${ }^{3}$ Grassé developed the concept of stigmergy during his study of termites. However, it has since been used and applied to other groups of social insects [28], not least in the study of ants [14]. Over the last decade or so the concept of stigmergy that the regulation of building activities among social insects does not depend so much on the workers themselves as on the nest structure. A stimulating configuration triggers a building action of a termite worker, transforming the configuration into another configuration that may in turn trigger another (possibly different) action performed by the same termite or any other worker in the colony [11]. Thus, work in the termite colony is partly coordinated by virtue of the individual termites acting on evidence of work accomplished previously by themselves or others.

Grassé used a stimuli-response model of action characteristic of the field of entomology in his work. We must be careful not to transpose this model of action to the context of human practice. CSCW is obviously dealing with humans rather than insects, so we have to leave Grassé's stimuli-response model of action behind. In the context of human practice and in relation to the concept of stigmergy perhaps we could ask: How does the comprehension of work accomplished previously lead to the coordination of cooperative work? Accounting for the process of comprehension could serve to sever the bond to any remaining stimuli-response model of action with regard to the concept of stigmergy. In addition, it could shed some light on how practices of stigmergy unfold in a human context.

The question above revolves around the concept of comprehension (verstehen) as Wittgenstein would have put it in his later writings. According to Wittgenstein [29] to comprehend something entails the ability to perform certain actions, verbal or non-verbal, in accordance with an established practice. For an architect, comprehending the CAD model of a building entails the ability to alter or elaborate the model. To comprehend the sounds of the words 'how many stories is the building?' entails the ability to answer for example 'it is twelve stories high'. Strictly speaking, that is all comprehension is. That is to say, comprehension is the ability to perform certain actions, verbal or non-verbal, in accordance with a practice $[29,30]$. We could suggest that stigmergy in a human context is based upon such comprehension. That is to say, with regard to human beings we could suggest that in the field of work some entity or other must be comprehended as 'evidence of work previously accomplished' in order for practices of stigmergy to unfold. In a human context it is a matter of comprehension, rather than stimuli and response.

These considerations aside (we shall return to the theme below), the central question is: does the concept of stigmergy add anything to our ability to account for coordinative practices in collaborative work? Perhaps this issue could be explored by comparing the concept of stigmergy to the wellestablished concepts within CSCW, namely articulation work [23] and feedthrough $[6,7]$.

has been introduced to the field of telecommunication especially in connection with the development of algorithms for network traffic, these algorithms are sometimes termed 'ant algorithms', [27] and to the field of robotics [8]. 


\subsection{Stigmergy Compared to Articulation Work}

Recall the concept of articulation work: all cooperative work practices entail activities that in a certain sense are aimed, not so much at the work itself, but rather at the cooperation typically: who does what when. Cooperative work is articulated in the sense that actors involved in cooperative work must share, allocate, coordinate, mesh, interrelate etc. their distributed individual activities [19, p.14]. This second order work is conceptualised as articulation work by Strauss [23]. An example of articulation work that springs to mind is the case of the assignment of the staff in the architectural office to specific tasks. In this case the employees of the architectural office are assigned to various tasks at a weekly meeting, each architect or building engineer is assigned to one or more of the building projects of the architectural office. This articulation work is done with the support of a sheet of paper upon which names of employees and building projects are matched for specified periods of time. The coordinative efforts performed using the task assignment sheet could be described as a kind of second order work, as articulation work performed in relation to the building projects.

Articulation work is often done with the support of coordination mechanisms such as the task assignment sheets mentioned above or meeting schedules, plans and forms. According to Schmidt \& Simone, a coordination mechanism is a construct consisting of, one the one hand, a coordinative protocol (an integrated set of procedures and conventions stipulating the articulation of interdependent distributed activities) and, on the other hand, an artifact in which the protocol is objectified [17, p.166]. In contrast to articulation work, practices of stigmergy does not rely on the use of coordination mechanisms - claiming so would be a contradiction in terms in the sense that there is no place for a coordinative protocol when coordination is achieved by acting directly on the evidence of work previously accomplished. We could suggest that the use of a coordination mechanism is evidence of a second order effort to coordinate work, an effort that there is no place (or need) for in practices of stigmergy.

Compared to the concept of articulation work, one could claim that stigmergy is not articulation work in the sense that stigmergy does not entail what could be considered extra work solely aimed at the coordination. In this view, we are stressing that stigmergy is a practice where collaborative work is coordinated on the basis of the evidence of work previously accomplished and not by way of meta work, supported by coordination mechanisms, aimed solely at the cooperation. If we employ this distinction, stigmergy is not articulation work. There is no discourse about work, no coordination mechanisms, and no coordinative protocols; in short there is no second order effort to achieve coordination in practices of stigmergy. Recall how the architect creates the outline of the building and the construction engineer creates the geometry of the concrete structure in a separate construction model based on that. Recall how, the sanitation specialist, for example, will take notice of the model for the concrete structure and seek to align the sanitation with it. Actors coordinate their cooperative efforts by acting on the evidence of work previously accomplished. It is partly the actors' repeated performance of stigmergy that contributes to the iterative process of designing a building. Here, the actors are simply doing their job, going about their business without making any second order effort to coordinate anything, and yet coordination of collaborative work is taking place. That is to say, in addition to relying on meetings, plans and schedules actors coordinate their cooperative efforts in practices of stigmergy: by acting directly on the evidence of work previously accomplished by themselves or others.

In a preliminary manner we could, perhaps, rest the distinction between articulation work and stigmergy on a distinction between coordination done with second order work (articulation work) and coordination achieved by directly acting on the evidence of work previously accomplished (stigmergy). This seems to be a tenable position to take, since it makes it possible to distinguish with relative clarity between two forms of coordination of cooperative work based on artifacts. It speaks in favour of the distinction between articulation work and stigmergy that, without it, we would be compelled to place two modes of coordination in the same category (as far as I can see). Seemingly, this could be avoided by upholding the distinction between articulation work and stigmergy.

\subsection{Stigmergy Compared to Feedthrough}

Leaving the distinction between articulation work and stigmergy for now (we shall return to it below), another concern appears. Perhaps other, more established concepts within $\mathrm{CSCW}$ and related research fields are already doing what Grassé's notion of stigmergy does. Are stigmergy and feedthrough, for example, interchangeable concepts? In addition to contrasting stigmergy with articulation work, perhaps it could also be helpful to contrast the concept of stigmergy with Dix's concept of feedthrough $[6,7]$. We shall do so for two reasons, in order to compare the concepts of stigmergy and feedthrough, and in order to approach a better understanding of how the comprehension of work accomplished previously leads to the coordination of cooperative work.

According to Dix in some cases cooperative work is coordinated through the artifact rather that by direct face-toface interaction or by other forms of verbal interaction. Dix states that:

'In a cooperative setting not only is it important to see one's own updates, but also to see the effects of other people's actions. This is feedthrough. The presence of feedthrough effectively creates an additional channel of communication through the artefacts themselves' [7, p.38].

According to Dix, this form of coordination is often more important than verbal direct communication. It is effective, partly because it is tied so closely to the work itself, and partly because it is implicit, unconsciously noted and acted upon. Perhaps we could note that so far Dix is describing a coordinative practice akin to stigmergy. Consider, however, Ramduny \& Dix [15] in a discussion of awareness of user activity in a collaborative environment:

'Delivering feedthrough at the wrong pace can be problematic. If it is too slow, users may have to act without up to-date knowledge of one another's actions. If it is too 
fast, users may be distracted by irrelevant changes. Some feedthrough is very goal-directed - information directly used by users in their tasks' [15, p.122].

The notion that feedthrough can be delivered at the "wrong pace' seems to indicate that in some instances the 'information' that feed through the artifacts is distinct from the efforts that is being coordinated. How else could it be delivered at the 'wrong pace'? It seems that, at least in some instances, the concept of feedthrough is concerned with 'metainformation' used to coordinate collaborative work.

Furthermore, the concept of feedthrough seems to rely on the notion that 'peoples actions' feed through the artifacts from actor A to actor B in the form of 'information'. Dix and Beale:

'The sharing of information comes because of feedthrough, when people are aware of and respond to the effects of one another's actions. In the sales situation the information from the factory floor must be timely, that is feedthrough of the factory staff's actions to the sales force.' $[6, \mathrm{p} .6]$

Perhaps a closer look at the concept of information is warranted. The scientific formulation of the concept of 'information' can be traced back to the 'mathematical theory of communication' developed shortly after WWII by Claude E. Shannon for the purpose of measuring the transportation capacities of communication networks [20, p.379]. Of course, the word 'information' was in common usage for many years before its scientific conceptualisation. It was recorded in print in 1390 to mean 'communication' or 'knowledge' or 'news' of some fact or occurrence (Oxford English Dictionary). However, as a part of his mathematical theory of communication, Shannon coined a definition of information that transformed it into a physical parameter capable of quantification. He accomplished this by separating information and meaning. He applied 'meaning' to the semantic part of a message and used 'information' to refer to the quantity of different possible messages that could be carried along a channel of communication at any one time depending on the length of the message and on the number of choices of symbols for transmission at each point in time [1, p.119]. For his purpose, this was quite appropriate, because semantic aspects of communication are irrelevant to the engineering problem [20, p.379]. Shannon had coined a quantitative concept to be used for measuring and emphasized that 'information' should not be confused with 'meaning' [21, p.8].

In relation to the concept of feedthrough, does the term 'information' refer to a quantitative measure, to meaning or both? Perhaps the very idea that information or some other fixed correlation between ideas and symbols migrate through the artifact is untenable. We could suggest that it is untenable in the sense that it presupposes the actor's efforts to comprehend the appearance of the artifacts (we shall return to this theme below).

Here we could pause and suggest that stigmergy and feedthrough are not interchangeable concepts, in the sense that the concept of stigmergy is not concerned with second order efforts to coordinate collaborative work and further more does not rely on the notion of information.
At this point we have tried to show that stigmergy is a concept distinct from the concepts of articulation work and feedthrough respectively. Perhaps it could prove to be a valuable addition to the $\mathrm{CSCW}$ conceptual toolkit in relation to the analysis and description of coordinative practices. In order to explore this matter further we could attempt to approach an understanding of how the comprehension of work accomplished previously leads to the coordination of cooperative work in practices of stigmergy. In the course of doing so we shall briefly consider what Roy Harris [13] has dubbed 'the language myth'. We shall do so in order to avoid associating our understanding of practices of stigmergy with it.

\section{THE LANGUAGE MYTH}

The influential linguist Roy Harris has coined the term 'the language myth' [13]. According to Harris, three assumptions are associated with the language myth - a myth Harris rejects. One is that in language actors somehow encapsulate their thoughts in the signs they use (and that these signs become information). Another is that the signs (or information) used have the property of containing thoughts in an invariant manner. Thirdly, when reading or listening actors 'extract' the thoughts from the signs (or information) in which they are encoded [13, p.12]. In Toolan's description, the language myth essentially regards communication as the 'faxing' of thoughts from actor $\mathrm{A}$ to actor $\mathrm{B}$, and the act of communication as being, in a manner of speaking, comparable to properly 'handshaking' fax machines [26]. According to Harris, such an understanding of language and communication is telementational in nature and leads to the following account of how human actors communicate by the use of artifacts: Suppose actor A has a thought that he wishes to communicate to actor B, for example, that glass is brittle. His task is to search among the sentences of a language known to himself as well as to actor $\mathrm{B}$, and select that sentence which has a meaning appropriate to the thought being conveyed; for example glass is brittle. He then encodes the sentence in its appropriate written form from which actor B is to decode it. By virtue of knowing what it means, actor B grasps the thought that actor A intended to convey to him, i.e. that glass is brittle $[13$, p.10]. Stated in this manner, it sounds like common sense; sounding like common sense is one of the powerful sources of appeal of the language myth according to Harris.

The language myth draws its appeal from being entrenched in the very words we use to describe communication. According to Reddy, the English language alone hosts more than a hundred expressions that are based on what he calls 'the conduit metaphor' [16]. Reddy calls it 'the conduit metaphor', because it implies that thoughts are transferred from actor A to actor B through some conduit or other. Reddy argues that it is almost impossible for an English speaker to discuss communication at all without committing to some form or other of that metaphor ${ }^{4}$.

\footnotetext{
${ }^{4}$ As a case in point consider again the concept of feedthrough. Is it based on one such conduit metaphor? Perhaps, to the extent that it suggests that information is fed from actor A to actor B through the conduit of artifacts (e.g. [15, p.122] \& [6, p.6]). If we accept that the analytical use of the concept of feedthrough is, in some instances, a commitment to a form of
} 
Following Harris and Reddy, we could suggest that it is not tenable to maintain that meaning can take on a fixed form (of for example information) and migrate through artifacts. That is to say, there is no conduit of a stable and meaningful entity that migrates through the artifacts. Perhaps we have to look for another understanding of the matters at hand in relation to practices of stigmergy.

\subsection{The Integrational Approach}

Harris proposes an alternative to the language myth, to the telementational approach and the idea of language as a fixed code. He offers the integrational approach, based on premises that differ from the language myth [12]. Perhaps this approach is useful for our purpose of exploring how the comprehension of work accomplished previously leads to the coordination of cooperative work in practices of stigmergy.

In contrast to the assumptions of the language myth, Harris states that the sign does not exist outside the context which gives rise to it: No abstract invariant remains 'the same' from one context to another [12, p.22]. Harris presents an example to clarify his position:

'In every day parlance the word sign often refers to a physical object, as for instance in the Highway Code to place a 'red warning sign' (a reflecting triangle) on the road at least 50 meters in front of a vehicle that has broken down ${ }^{5}$. This use of the word sign is a potential source of confusion. For the integrational theorist, the reflecting triangle does not become a sign until it is appropriately placed in a situation of the kind described. The same physical object - the red triangle - was not a sign during the time it remained in the boot of the motorist's car in readiness for such an emergency; nor having once functioned as a sign, will it continue to do so when the motorist eventually puts it back in the boot again and proceeds on the journey. The spatiotemporal continuity of the object is irrelevant to its semiological role' [12, p.53].

In the integrational approach the sign only exists in the situation that gives rise to it, whereas the telementational approach views the sign as a stable entity. The understanding of the sign as a stable entity can lead to the view that artifacts somehow hold information: a person formulates a thought and records it in an artifact as information that another person later receives during his/her use of the artifact.

With regard to the concept of stigmergy we could adopt the integrational approach ${ }^{6}$ : an understanding of the sign as only existing in the situation that gives rise to it. The material appearance of the artifact can give rise to meaningful signs given an appropriate context of practice. That is to say, in

the conduit metaphor, this associates the concept of feedthrough with the language myth.

5 The Highway Code, rev. ed. (London: HMSO, 1987), art. 133.

${ }^{6}$ This is done at our own peril, please do not blame Grassé [11] practices of stigmergy the meaning of signs or marks on artifacts is relative to a context, such as architectural practice, and does not exist outside it.

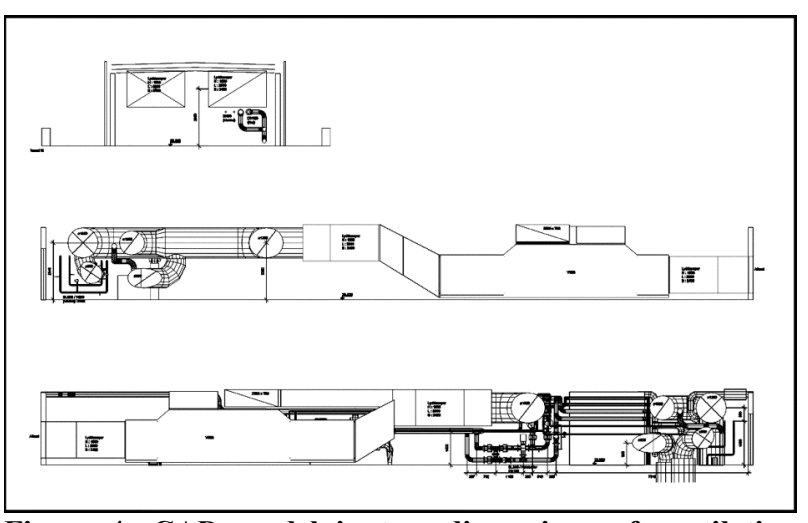

Figure 4: CAD model in two dimensions of ventilation system seen from different angles.

As we have observed above, in a building project with numerous other actors the engineer, for example, adds ventilation elements to a halfway-completed CAD model created by someone else. Here, he or she comprehends the signs in the CAD model as meaningful by virtue of the context. That is to say, when he or she adds ventilation elements, intake, pipes, exhaust etc. (see figure 4), to a model of a building, this action is relative to the context of architectural practice, including the nature of the building project, the division of labour among the work ensemble, the progress of the building project, the task at hand etc. All these contextual clues inform the actor in question how to comprehend the signs he or she finds on CAD models made by others, and how to act on these signs in relation to the task at hand. To an outsider the model hardly makes much sense, one could imagine. Here we are dealing with the comprehension of integrational signs, rather than the migration of information, or as we have discussed earlier, stimuli-response action. This discussion also relates to a former issue, that is, the origin of the concept of stigmergy in the field of entomology and the associated stimuli-response model of action. Perhaps accounting for the process of comprehension following the integrational approach outlined above could serve to sever the bond to any remaining stimuli-response model of action in regard to the concept of stigmergy.

Following the integrational approach outlined above, we could suggest that stigmergy (acting on the evidence of work previously accomplished) amounts to acting on the comprehension of integrational signs. In other words, acting on marks in relation to their context, the field of work they are found in, is constitutive of practices of stigmergy. In this manner we could attempt to divorce the concept of stigmergy from a stimuli-response notion of action. This way, we would have left the world of the non-human animals behind and entered the realm of human work practice.

We could suggest resting the concept of stigmergy on a notion that signs left or modifications made by individuals on artifacts may, given an appropriate context of practice, become meaningful to these individuals themselves or to others and in turn inspire new actions on artifacts. Thus, stigmergy 
understood as a form of coordination of cooperative work with artifacts cannot tenably be described as involving the migration of information from one actor through an artifact to another actor - that would be telementation.

This does not necessarily entail that actors make a great effort to make sense of the appearance of artifacts. According to Bourdieu [3] practice can entail a fair amount of unreflective routine and repetition. Most of the time, making sense requires no effort for the skilled actor, but for the novice this can be a different matter. What is meaningful from the viewpoint of the individual actor is closely connected to the actor's knowledge of and experience with the work setting and ultimately the world in which the actor is born. In making sense of the appearances of artifacts, an actor draws on his/her knowledge of the work setting, the tools, the conventions and concepts, the normal routines and procedures, and ultimately on general culture.

We have now attempted to outline a general notion of stigmergy [11] that is in part distinct and meaningful by virtue of being contrasted with and differentiated from the concept of articulation work $[23,19]$ and the concept of feedthrough [6, 7]. In regard to the concept of articulation work, the concept of stigmergy could be viewed as a complementary rather than as an interchangeable concept, in the pursuit of an understanding of coordinative practices in cooperative work. Compared to the concept of articulation work, stigmergy is not articulation work in the sense that stigmergy does not entail what could be considered second order efforts aimed at the coordination. In this view, we are stressing that stigmergy is practice, where collaborative work is coordinated based on the evidence of work previously accomplished and not by way of meta-work aimed solely at the cooperation. In connection to the concept of feedthrough the above discussion suggests that stigmergy and feedthrough are not interchangeable concepts. In the sense that the concept of stigmergy is not concerned with second order efforts to coordinate collaborative work and furthermore does not rely on the notion of information.

In sum, following Grassé [11] perhaps we could suggest the following description of the concept of stigmergy: Some instances of cooperative work are coordinated by virtue of the evidence found on artifacts of work previously accomplished: Signs left or modifications made by individuals in the field of work, given an appropriate context of practice, become meaningful to these individuals themselves or to others and in turn inspire new actions on artifacts.

Having considered the concept of stigmergy especially in regard to the concept of articulation work, we will now attempt to answer the previously stated question: does the concept of stigmergy add anything to our ability to account for coordinative practices in collaborative work? One the one hand, speaking against the introduction of the concept of stigmergy to the analysis of coordinative practices is the claim that it is superfluous, that the field of CSCW and related research areas can do without it. One the other hand, as mentioned above, it speaks in favour of the distinction that, without it, we would be compelled to conflate coordination done with second order work, one the one hand, with coordination achieved by directly acting on the evidence of work previously accomplished, on the other hand. Perhaps only further empirical research can settle whether the concept of stigmergy has a place in the analysis of coordinative practices. However, the previous descriptions of coordinative practices in architectural work indicate that the analysis of coordinative practices in collaborative work could benefit from the concept of stigmergy.

\section{CHALLENGES FOR CSCW IN RELATION TO THE DISTINCTION BETWEEN STIGMERGY AND ARTICULATION WORK}

If we accept the distinction between articulation work and stigmergy, perhaps we can also differentiate between coordination mechanisms in the service of articulation work and representational artifacts associated with practices of stigmergy.

As mentioned above, according to Schmidt \& Simone, a coordination mechanism is a construct consisting of a coordinative protocol (an integrated set of procedures and conventions stipulating the articulation of interdependent distributed activities) on the one hand and on the other hand an artifact in which the protocol is objectified [17, p.166]. It is a resource for action that reduces the complexity of articulating cooperative work by providing a means which actors can rely on to provide a valid and yet limited set of options for coordinative action in any given situation [17, p.174]. Recall the task assignment sheet described above, perhaps we could describe it as one such coordination mechanism. In this case the employees of the architectural office are assigned to various tasks at a weekly meeting, each architect or building engineer is assigned to one or more of the building projects of the architectural office. This articulation work is done with the support of a sheet of paper upon which names of employees and building projects are matched for specified periods of time. If we consider the work on the building projects the primary task, the coordinative efforts performed could be described as a kind of second order work, as articulation work supported by a coordination mechanism, i.e. the task assignment sheet. In contrast to articulation work, practices of stigmergy do not rely on the use of coordination mechanisms, there is no place for a coordinative protocol when coordination is achieved by acting directly on the evidence of work previously accomplished. Perhaps observations of the use of a coordination mechanism are empirical evidence of a second order effort to coordinate work, an effort that there is no place and no need for in practices of stigmergy. This brings us to a discussion of a possible distinction between representational artifacts associated with practices of stigmergy and coordination mechanisms associated with articulation work. Is such a distinction of any analytical value - perhaps it is, if indeed we do more than merely name objects.

According to Gibson, the arbitrary names we give objects have no connection to what they afford [10]. The fact that a stone can be a missile does not imply that it cannot be other things as well. It can serve as a paperweight, a hammer, or a bookend. It can be piled on top of other stones and become a wall of stones. To perceive the affordances of an object is not tantamount to classifying it according to some scheme of names. If you know what an object can be used for, you can 
call it whatever you like [10, p.134]. It is, we might add, the act of knowing the practices of the artifact that reveals its affordances. This is emphasized in order to avoid the, as Gibson sees it, fruitless exercise of assuming fixed classes of objects, each defined by a common feature and then given a name [10]. This seems to be in resonance with Wittgenstein: you cannot specify the sufficient features of a class of objects to which a shared name is given. In many cases a name can only indicate a 'family resemblance' [30].

Following Wittgenstein, rather than attempt to specify the sufficient features of a class of objects and give it a shared name, we could attempt to delineate two families of artifacts that are inconceivable apart from the respective practices that in part constitute them. That is, we could attempt to do more than merely name objects. Schmidt and Wagner distinguish between representational artifacts and coordinative artifacts:

Although they all invoke and incorporate various conventional practices and, in the case of the CAD plan, at times even highly formalized ways of graphical communication, representational artifacts are subservient to their function; namely, to represent. ... The coordinative artifacts, by contrast, are not in the service of depicting the material world or, indeed, of depicting anything. They are in the service of imposing order. [18, p. 395]

Here we could interject that the function of representational artifacts is not only to represent, as acknowledged by Schmidt and Wagner. They also play a key role in the integration of cooperative work as the corner stone of practices of stigmergy. However, that is not the main point here. The point is that perhaps one of the future challenges for $\mathrm{CSCW}$ will be the question of whether a tenable distinction can be made between two 'families' of artifacts (to use Wittgenstein's term) imbedded in their respective practices. We would suggest a distinction between on the one hand the representational artifacts associated with practices of stigmergy and on the other hand the coordinative artifacts associated with articulation work. These distinctions could be explored further for the benefit of the design of computer support for cooperative work.

\section{ACKNOWLEDGMENTS}

I am indebted to Morten Zinglersen and the staff of Thora Arkitekter $\mathrm{A} / \mathrm{S}$ in Copenhagen for giving me access to their work. Furthermore, I am grateful to Lisbeth Cederholm and Jan Søgaard of Leif Hansen Rådgivende Ingeniører A/S for letting me take up so much of their time. Furthermore, the comments and opinions of Kjeld Schmidt are gratefully acknowledged. In addition, the reviewer's helpful comments and suggestions are greatly appreciated. Lastly thanks to Morten Visby for proof reading.

\section{REFERENCES}

[1] Aspray, William. The Scientific Conceptualization of Information: A Survey, Annals of the History of Computing 7:2: 117-140, 1985.
[2] Bardram, J. E. and Bossen, C. A web of coordinative artifacts: collaborative work at a hospital ward. In Proceedings of the 2005 international ACM SIGGROUP Conference on Supporting Group Work (Sanibel Island, Florida, USA, November 06 - 09, 2005). GROUP '05. ACM Press, New York, NY, 2005.

[3] Bourdieu, Pierre: Outline of a Theory of Practice, Cambridge University Press, Cambridge, 1977.

[4] Carstensen, Peter H. and Carsten Sørensen (1996): From the social to the systematic: Mechanisms supporting coordination in design. Computer Supported Cooperative Work. The Journal of Collaborative Computing, vol. 5 , no. 4, 1996, pp. 387-413.

[5] Divitini, Monica, Carla Simone, and Kjeld Schmidt. ABACO: Coordination mechanisms in a multi-agent perspective. COOP'96: Second International Conference on the Design of Cooperative Systems, Antibes-Juan-lesPins, France, 12 - 14 June, 1996. INRIA Sophia Antipolis, France, pp. 103-122.

[6] Dix, A and R. Beale. Introduction. In Remote cooperation: CSCW issues for mobile and tele-workers, Eds. A. J. Dix and R. Beale. Springer Verlag. pp. 1-10, 1996.

[7] Dix, A. Challenges for Cooperative Work on the Web: An analytical approach. Computer-Supported Cooperative Work: The Journal of Collaborative Computing, vol. 6, pp. 135-156, 1997.

[8] Dorigo, M., E. Bonabeau, and G.Theraulaz. Ant Algorithms and Stigmergy. Future Generation Computer Systems, 16(8):851-871, 2000.

[9] Francoeur, E and J. Segal. From Model Kits to Interactive Computer Graphics. In Models - The Third Dimension of Science, Eds. S. de Chadarevian \& N. Hopwood, Stanford University Press, Stanford, pp. 402 - 429, 2004.

[10] Gibson, J.J. The Ecological Approach to Visual Perception, New Jersey \& London, Lawrence Earlbaum Associates, 1986.

[11] Grassé, P.P. La reconstruction du nid et les coordinations inter-individuelles chez Bellicositermes natalensis et Cubitermes sp. La théorie de la stigmergie: Essai d'interprétation des termites constructeurs, Insect Sociaux, vol. 6, pp. 41- 83, 1959.

[12] Harris, Roy. Signs of Writing, Routledge, London and New York, 1995.

[13] Harris, Roy. The Language Myth. Duckworth, London, 1981.

[14] Hölldobler B. and Wilson E.O. Journey to the Ants. Bellknap Press/Harvard University Press, 1994.

[15] Ramduny-Ellis D. and A. Dix. Impedance Matching: When you need to know What. Proceedings of HCI2002 (London, September 2002). Faulkner X., Finlay J., 
Detienne F. (eds.), London, UK, Springer, pp 121-137, 2002.

[16] Reddy, M.J. The conduit metaphor - a case of frame conflict in our language about language. In A. Ortony (Ed.), Metaphor and thought Cambridge: Cambridge University Press, pp. 284-297, 1979.

[17] Schmidt, Kjeld \& Carla Simone. Coordination mechanisms: Towards a conceptual foundation of CSCW systems design, Computer Supported Cooperative Work: The Journal of Collaborative Computing, vol. 5, no. 2-3, 1996, pp. 155-200

[18] Schmidt, Kjeld and Ina Wagner. Ordering systems: Coordinative practices and artifacts in architectural design and planning, Computer Supported Cooperative Work (CSCW): The Journal of Collaborative Computing, vol. 13 , no. 5-6, 2004, pp. 349-408.

[19] Schmidt, Kjeld and Liam J. Bannon. Taking CSCW seriously: supporting articulation work', Computer Supported Cooperative Work (CSCW): An International Journal, vol. 1, no. 1-2, pp. 7-40, 1992.

[20] Shannon, Claude E. A mathematical theory of communication, The Bell System Technical Journal, vol. 27, July, October 1948, pp. 379-423, 623- 656.

[21] Shannon, Claude E. and Warren Weaver. The Mathematical Theory of Communication, University of Illinois Press, Urbana, 1949.
[22] Simone, Carla and Monica Divitini. Ariadne: supporting coordination through a flexible use of the knowledge on work processes. Journal of Universal Computer Science, vol. 3 , no. 8,1997 , pp. 865-898.

[23] Strauss, Anselm. Work and the division of labor. The Sociological Quarterly, vol. 26, no. 11985, pp. 1-19, 1985.

[24] Theraulaz, G., Bonabeau, E. A brief history of stigmergy. Artificial Life 5 (1999) 97 - 116.

[25] Turnbull, David. The ad hoc collective work of building Gothic cathedrals with templates, string, and geometry, Science, Technology, \& Human Values. vol. 18, no. 3, Summer 1993, pp. 315-340.

[26] Toolan, M. A Few Words on Telementation. Language Sciences, vol. 19. No. 1 1997, pp. 79-91.

[27] White, T and Bernard Pagurek. Towards multi-swarm problem solving in networks. In Proceedings of Third International Conference on Multi-Agent Systems (ICMAS'98), pages 333-340, 1998.

[28] Wilson, E.O. Sociobiology: The New Synthesis, Cambridge, MA: Belknap Press, 1975.

[29] Wittgenstein, L. On Certainty. Blackwell Publishing, Oxford, 1975.

[30] Wittgenstein, L. Philosophical Investigations. Blackwell Publishing, Oxford, 2001. 